\title{
Clinical Evaluation of 6-Month Efficacy of $40 \%$ in-Office Whitening Treatment
}

\author{
Amar Piknjač1@i], Dubravka Knezović Zlatarić2@i]
}

'Private Dental Office Floss\&Gloss, Sarajevo, Bosnia and Herzegovina.

${ }^{2}$ Department of Removable Prosthodontics, School of Dental Medicine, University of Zagreb, Zagreb, Croatia.

Correspondence: Piknjač Amar, Private Dental Office Floss\&Gloss, Maršala Tita 5, 71000 Sarajevo, Bosnia and Herzegovina. E-mail: amar.piknjac@,gmail.com

Academic Editor: Alessandro Leite Cavalcanti

Received: 09 November 2020 / Review: 20 January 2021 / Accepted: 02 February 2021

How to cite: Amar P, Zlatarić DK. Clinical evaluation of 6-month efficacy of $40 \%$ in-office whitening treatment. Pesqui Bras Odontopediatria Clín Integr. 2021; 21 :e0236. https://doi.org/10.1590/pboci.2021.095

\begin{abstract}
Objective: To assess the 6-month efficacy of natural tooth color change in in-office bleaching treatment in terms of time by using a spectrophotometer. Material and Methods: A total of 20 participants were chairside treated with $40 \%$ hydrogen peroxide (HP), three applications each 20 minutes, in one appointment. Instrumental color measurement was performed on six anterior maxillary teeth before bleaching (baseline - $t_{0}$ ), immediately after in-office bleaching and rehydration of the teeth $\left(t_{1}\right), 3$ months $\left(t_{2}\right)$, and 6 months after bleaching treatment $\left(t_{3}\right)$. The spectrophotometer measured the tooth shades based on the CIE $\mathrm{L}^{*} \mathrm{a} \mathrm{b}^{*}$ color notation system and Bleach index during the period of observation. CIE $\mathrm{L}^{*} \mathrm{a} * \mathrm{~b} *\left(\Delta \mathrm{E}_{\mathrm{ab}}\right)$ color differences were calculated. Results: The color change at $\mathrm{t}_{1}$ was $\Delta \mathrm{E}_{\mathrm{ab}}=3.2$, at $\mathrm{t}_{2}$ was $\Delta \mathrm{E}_{\mathrm{ab}}=1.8$, at $\mathrm{t}_{3}$ was $\Delta \mathrm{E}_{\mathrm{ab}}=1.2$ and overall color change of in-office method was $\Delta \mathrm{E}_{\mathrm{ab}}=3.6(\mathrm{p}<0.05)$. A significant effect for the mean CIEL*a*b* values was detected as within time $b^{*}$ values decreased significantly $(\mathrm{p}<0.05)$. Bleach index values significantly decreased during the time of observation, too $(\mathrm{p}<0.05)$. Conclusion: The in-office bleaching treatment using $40 \%$ hydrogen peroxide was effective, and the results showed a statistically significant decrease in color change during the period of 6-month observation.
\end{abstract}

Keywords: Esthetics, Dental; Tooth Bleaching; Tooth Bleaching Agents; Hydrogen Peroxide. 


\section{Introduction}

Physical attractiveness plays an important role in everyday life, and dental appearance is part of it. The significant factors affecting overall dental appearance are shape and position of tooth, color, general arrangement of the dentition, the quality of restoration, and tooth color as one of the most critical factors determining satisfaction with dental appearance [1]. Self-satisfaction with tooth color decreases with increasing severity of discoloration [2]. White teeth have been positively correlated with high ratings of social competence, intellectual ability, psychological adjustment and relationship status [3]. Treatment options to whiten teeth can vary depending on the cause of the discoloration and may include: in-office or at-home bleaching, micro abrasion or macroabrasion, composite bondings, veneers, crowns [4-6].

Tooth bleaching is a conservative and very effective method to change the color of the teeth and has been practiced in dentistry for many years [7,8]. It involves an improvement in the lightness of the teeth in a short time, appears not invasive for the patients, and therefore it is ideal for young people who have healthy unrestored teeth and healthy gums [9].

According to American Dental Association (ADA), there are three fundamental approaches for bleaching vital teeth: in-office or power bleaching, at-home or dentist-supervised night-guard bleaching, and bleaching with over-the-counter (OTC) products [10-12]. The current in-office bleaching technique typically uses different concentrations of hydrogen peroxide (HP), between $15 \%$ and $40 \%$ (depending on the country's regulations), with or without light and in the presence of rubber dam isolation [13]. Here, the dentist has complete control throughout the procedure and has the ability to stop it when the desired shade/effect is achieved. The in-office treatment can result in significant bleaching after only one treatment, but many more may be needed to achieve an optimum result [14]. Regardless of the technique or product used, the mechanism of action of bleaching agents is based on a complex oxidation process with the release of reactive oxygen species, which penetrate through the pores of enamel rods and reach the dentin, breaking down organic molecules and producing lighter, smaller, and clearer compounds [15].

The purpose of this prospective, randomized clinical study was to assess the 6-month efficacy of natural tooth color change in in-office bleaching treatment in terms of four-time points by using a spectrophotometer. The null hypothesis was that no differences would be found in dental color before and after the treatment.

\section{Material and Methods}

Study Design and Observer's Calibration

Two observers in this in vitro study underwent testing of their color assessment ability, intra- and inter-observer reliability before the clinical measurement. For testing their color assessment ability, Farnsworth-Munsell 100 HueColor Vision Test was used [16]. Each observer measured the L value (lightness) of the right maxillary central incisal in four patients two times with the interval of 5 minutes (12.00 am), in the room with dental light and natural light source (dental office) (light temperature $6500 \mathrm{~K}$, illuminance 1000 lux) using intraoral spectrophotometer VITA Easyshade Advance 4.0 ${ }^{\circledR}$ (VITA Zahnfabrik, Bad Sackingen, Germany) in "tooth single" mode. Two-color measurements of each observer were compared mutually.

Sample

In previous research, supported by University of Zagreb in 2014 - BM1.57 (this study is part of it) analyzed the statistical power and confirmed the adequate sample size between 10 and 120 subjects [17]. 
A total of 20 participants were enrolled in the study, seventeen women and three men (mean 31,2 and 31,6 years; respectively), according to the inclusion and exclusion criteria described in Table 1. Dropouts were replaced with new participants to increase the power of the statistical analysis. All the participants wanted to change the color of their teeth for cosmetic reasons.

Table 1. Criteria used in this study.

\begin{tabular}{|c|c|}
\hline Inclusion Criteria & Exclusion Criteria \\
\hline Adult (18-70 years) & Presence of tooth sensitivity \\
\hline $\begin{array}{l}\text { Minimum of } 20 \text { natural teeth, including incisors and } \\
\text { canines in both arches }\end{array}$ & $\begin{array}{l}\text { Currently undergoing treatment for caries, gingivitis, or } \\
\text { periodontitis, or having undergone or currently } \\
\text { undergoing orthodontic treatment }\end{array}$ \\
\hline Willing to sign a consent form & $\begin{array}{l}\text { Structural alteration of tooth structure, or alternation of } \\
\text { oral mucosa }\end{array}$ \\
\hline Willing to return for post-whitening evaluation & $\begin{array}{l}\text { A medical condition that the investigator believed might } \\
\text { compromise the study or the individual's safety }\end{array}$ \\
\hline Good oral hygiene & Existing dental restorations in teeth to be whitened \\
\hline \multirow[t]{3}{*}{ No caries on teeth to be whitened } & Use of tobacco products during previous 30 days \\
\hline & Pregnant or breast-feeding women \\
\hline & Tetracycline-stained teeth or fluorosis \\
\hline
\end{tabular}

\section{Positioning Jig}

The participants' teeth were professionally cleaned - Proxyt RDA 36 (Ivoclar Vivadent AG, Schaan, Liechtenstein). One alginate - Hydrogum 5 (Zhermack SpA, Badia Polesine, RO, Italy) impression of the maxillary arch was made, and a stone model - Fuji rock EP Classic line (GC Corp., Tokyo, Japan) was produced to fabricate a modified form of bleaching tray, $1 \mathrm{~mm}$-thick thermoplastic tray material and thermoforming unit (Erkodent Erich Kopp GmbH, Pfalzgrafenweiler, Germany), to make a positioning jig to ensure placement of the tip of the spectrophotometer in the same position at every color measurement. An impression of the tip of the probe of the spectrophotometer was made and a cast was fabricated. The spectrophotometer probe cast was used as a stamp guide to mark the bleaching tray. The facial middle-third of the maxillary teeth was marked with the spectrophotometer tip cast using an ink pad. The facial marks were cut, leaving an opening for placement of the spectrophotometer probe. Prior to color measurement, the custom jig was positioned in the patient's mouth, and the spectrophotometer probe was positioned into the jig opening. The subjects also received a toothpaste - Opalescence Bleaching Toothpaste (Ultradent Products Inc., Salt Lake City, UT, USA) and a soft-bristled manual toothbrush (Oral-B Laboratories Inc., Iowa City, IA, USA). They were asked to brush at least twice a day to maintain a standardized home care regimen.

\section{In-office Bleaching Treatment}

Participants were chairside treated with 40\% HP - Opalescence Boost: PF 40 (Ultradent Products Inc., Salt Lake City, UT, USA). Prior to treatment, protective lip cream, a light-cured resin barrier OpalDam (Ultradent Products Inc., South Jordan, UT, USA) for gingival protection were applied (included in the kit). To prevent saliva from flowing through embrasures of anterior teeth, a saliva ejector and cotton rolls were used in the sublingual region. An expanded lip retractor was used to protect lips. An Ultradent IsoBlock (Ultradent Products Inc., South Jordan, UT, USA) was used to keep the tongue in a safe position. For Opalescence Boost PF, the activator was mixed into the bleaching agent using the proper syringe. The mixture was then applied 1-2 mm thick on the buccal surfaces of the teeth (canine to canine) of maxillary arch and remained on for twenty minutes, three applications. No heat or special lamps were used to complete the 
process. The agent was removed using suction and gauze only for a new application. After the third last application, teeth were rinsed with water and the gingival isolation and lip retractor were removed. Both the participants and the dentist wore protective glasses during the treatment.

\section{Instrumental Color Measurement}

Instrumental color measurement was performed on six anterior maxillary teeth before bleaching (baseline- $\mathrm{t}_{0}$ ), immediately after in-office bleaching and rehydration of the teeth $\left(\mathrm{t}_{1}\right), 3$ months $\left(\mathrm{t}_{2}\right)$, and 6 months after bleaching treatment $\left(t_{3}\right)$. The VITA Easyshade Advance 4.0 (VITA Zahnfabrik H. Rauter GmbH \& Co. KG, Bad Sackingen, Germany) spectrophotometer was used and calibrated according to the manufacturer's instructions, with the operating mode set to single shade measurement. To provide accurate repositioning of the spectrophotometer probe and measurement of the middle third of the labial tooth surface over time, a custom positioning jig was made for each participant, as described in the previous chapter. The spectrophotometer measured the tooth shades based on the CIE L*a*b* color notation system and Bleach index (BI). CIE L*a*b* $\left(\Delta \mathrm{E}_{\mathrm{ab}}\right)$ color differences were calculated [18]. Repeatability, accuracy and reliability of this device have already been tested and proved [19,20]. In 50\% initial color was A1 and in 50\%, A2 or darker.

\section{Statistical Analysis}

Data were statistically analyzed using the statistical program SPSS 19.0 (IBM SPSS, Chicago, IL, USA). To estimate intra-observer reliability paired t-test was used and to estimate inter-observer reliability ttest for independent samples was used. In both cases, the ICCs were calculated for both measurements. Normality of distribution was evaluated using Kolmogorov-Smirnov test.

A repeated-measures ANOVA was used to determine whether there were any differences in mean CIE $L^{*} a^{*} b^{*}$ values and BI values at four different time points in in-office bleaching group $\left(t_{0}, t_{1}, t_{2}, t_{3}\right)$ and Bonferroni corrected post hoc tests (paired t-tests) were conducted to explore the differences within the time points. The same statistical analysis was applied for differences in mean $\Delta \mathrm{E}$ values at three different time points $\left(\mathrm{t}_{1}\right.$ - immediately after the treatment, $\mathrm{t}_{1}-$ after 3 months, and $\mathrm{t}_{2}-$ after 6 months).

\section{Results}

Data were distributed normally and therefore parametric tests were performed $(\mathrm{p}>0,05)$. For both observers, the total error score in testing the color assessment ability using Farnsworth-Munsell 100 HueColor Vision Test (TES) was 21 and 22, meaning that they both had excellent ability of color assessment with error scores within the normal range (total error score 46 upward is considered as deviation) [16].

The results of paired t-test and t-test for independent samples for measurements of lightness on the right maxillary central incisor in four patients two times with the interval of 5 minutes in this study revealed no statistically significant differences for both observers $(\mathrm{p}>0.05)$. The high ICC values prove the observers' consistency too - intra-observer reliability for the first observer was 0.73 , and for the second 0.74 ; interobserver reliability for the first measurement was 0.89 , and for the second was 0.91 ( $\mathrm{p}<0.001)$.

Since it was proved that intra- and interobserver reliability in this study were high, mean values for CIE L* $\mathrm{C}^{*} \mathrm{~h}^{*}$ values measured by the observer reached higher ICC values were used in the further analysis. Twenty of 22 participants completed this study. Two were excluded because of the sensitivity they felt during in-office treatment, which was the reason for aborting the procedure.

The statistical analysis showed that, concerning the comparison of in-office bleaching procedures in four different time points, $\mathrm{L}^{*} \mathrm{a}^{*} \mathrm{~b}^{*}$ values, and Bleach index changed over time (Figures 1 and 2; Table 2). 
The mean $\mathrm{L}^{*}$ values reached the highest peak immediately after applying $40 \% \mathrm{HP}\left(\mathrm{t}_{1}\right)$ and maintained at that level over the observed period of 6 months. The mean $a^{*}$ values decreased slightly immediately after the treatment, but in a period of 3 months, returned to baseline value and remained so. The mean $b^{*}$ values remained almost unchanged after the bleaching treatment, but decreased significantly in 3 months $\left(t_{1}-t_{2}\right)$ and then remained again on that level till the end of the study. The mean Bleach index values revealed the same trend like $b^{*}$ values and significantly decreased in the period from $t_{1}$ and $t_{2}$ (Figure 1 and Table $2 ; \mathrm{p}<0.05$ ).

The mean CIEL*a*b* color change $\left(\Delta \mathrm{E}_{\mathrm{ab}}\right)$ values in in-office bleaching constantly decreased during the time of observation and are shown in Figure 2. Immediately after the bleaching treatment and rehydration of the teeth, $\Delta$ Eab was 3.2 in the next 3 months, decreased to 1.8, and continued to decrease further to 1.2 until the end of the study. The overall color change was 3.6, and it was found to be significant (Figure 2 and Table 2 ; $\mathrm{p}<0.05)$.

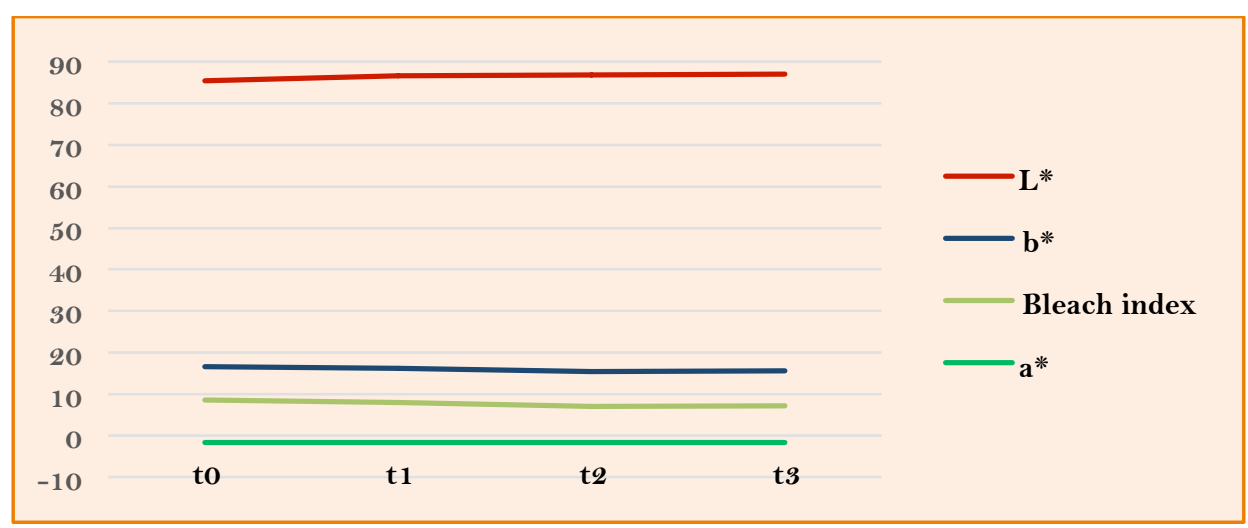

Figure 1. Mean $L^{*} a^{*} b^{*}$ and Bleach index values for in-office Boost $40 \%$ bleaching in time points observed.

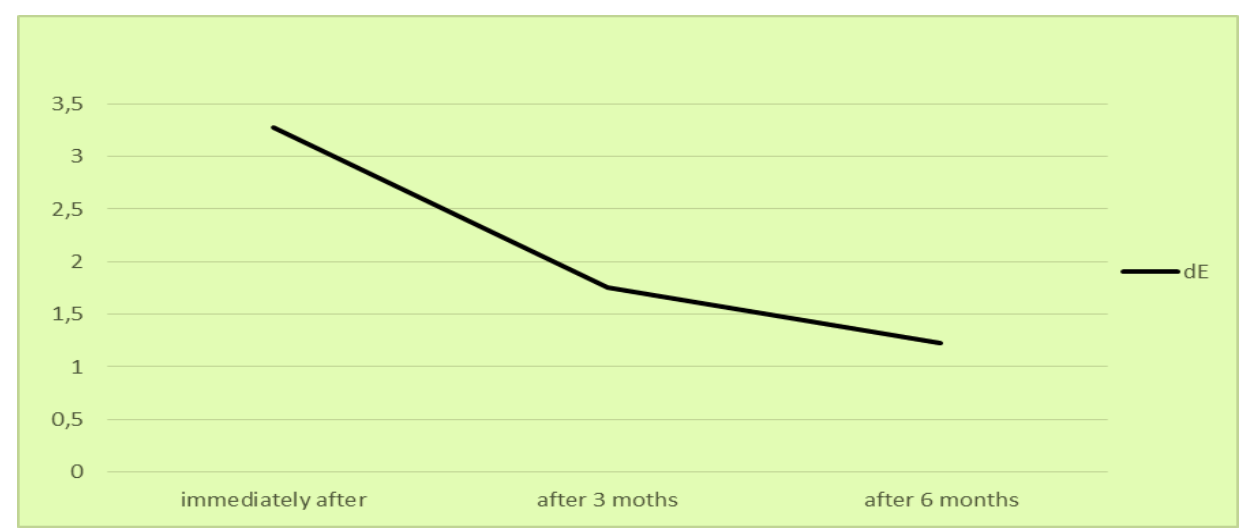

Figure 2. Mean $\Delta \mathrm{E}$ values for in-office Boost $40 \%$ bleaching in time points observed.

Table 2. Significance of the differences ( $F$ and p-values) in mean $L^{*} a^{*} b^{*}$, Bleach index (BI) and $\Delta E$ values $(x)$ and their standard deviations $(\mathrm{SD})$ in time points observed.

\begin{tabular}{cccccc}
\hline Independent Variables & Time Point & $\mathbf{x}$ & SD & F & p-value \\
\hline L $^{*}$ & to & 85.4 & 2.4 & 1.811 & 0.183 \\
& t1 & 86.6 & 2.9 & & \\
t2 & 86.8 & 2.8 & & \\
& t3 & 86.9 & 2.6 & &
\end{tabular}




\begin{tabular}{|c|c|c|c|c|c|}
\hline$a^{*}$ & to & -1.6 & 1.0 & 1.570 & 0.233 \\
\hline & $\mathrm{t} 1$ & -1.4 & 1.7 & & \\
\hline & $\mathrm{t} 2$ & -1.7 & 0.9 & & \\
\hline & $\mathrm{t} 3$ & -1.7 & 1.5 & & \\
\hline \multirow[t]{4}{*}{$b^{*}$} & to & 16.4 & 4.1 & 6.063 & 0.005 \\
\hline & $\mathrm{t} 1$ & 16.2 & 3.8 & & \\
\hline & t 2 & 15.4 & 3.9 & & \\
\hline & $\mathrm{t} 3$ & 15.5 & 3.9 & & \\
\hline \multirow[t]{4}{*}{ BI } & to & 8.7 & 3.8 & 32.326 & 0.000 \\
\hline & $\mathrm{t} 1$ & 8.0 & 3.5 & & \\
\hline & t 2 & 7.0 & 3.3 & & \\
\hline & $\mathrm{t} 3$ & 7.1 & 3.4 & & \\
\hline \multirow[t]{4}{*}{$\Delta \mathrm{E}$} & & & & 9.752 & 0.001 \\
\hline & $\mathrm{t} 1$ & 3.3 & 1.9 & & \\
\hline & $\mathrm{t} 2$ & 1.8 & 1.3 & & \\
\hline & $\mathrm{t} 3$ & 1.2 & 1.1 & & \\
\hline
\end{tabular}

\section{Discussion}

The null hypothesis was rejected as mean $\Delta \mathrm{E}_{\mathrm{ab}}$ values in in-office bleaching, upon completion of the observation (6 months), significantly differed from the baseline measurements (Figure 2 and Table 2 ; p $<0.05$ ). The result of our study is hard to compare with others because of different concentrations of materials used, different protocols and inconsistent monitoring periods in researches. Overall color change of in-office method in this six-month follow-up study using $40 \% \mathrm{HP}$ was $\Delta \mathrm{E}_{\mathrm{ab}}=3.6$.

Al Shethri et al. [21] using the same material but different protocol (two appointments, each with three 10 minute applications, in a period of 7 days), found mean $\Delta \mathrm{E}_{\mathrm{ab}}$ following second bleaching treatment reached 2.45, with the color relapse up to the fifth week and then remained stable. In our study, overall color change, as well as the change immediately after in-office bleaching and rehydration, were higher, but we found this color relapse to be a trend in 6-month follow-up, where $\Delta \mathrm{E}_{\mathrm{ab}}$ constantly decreased to reach 1.2 (Figure 2 and Table 2; $<<0.05)$.

Al Shetheri et al. [21] found the color stabilized by the fifth week, which was not found in our study. This could be attributed to a different protocol because they whitened twice with the weekly interval and we did it once conducting the manufacturer's instructions.

Klarić et al. [22] used the same material as we did and measured the color change immediately after the treatment, 1 and 6 months after. They found color changes to be much higher than ours, with $\Delta \mathrm{E}_{\mathrm{ab}} 1$ month after the treatment to be 7.1 and overall after 6 months 7.3 [22]. This can be explained by the fact that in their study, the initial tooth color was darker (31\% B3; 20\% A2) while in ours it was higher - 50\% of the participants had A1, and 50\% A2 or darker with mean L and b values to be 85,4 and 16,4, respectively (which was relatively high $\mathrm{L}$ and low $\mathrm{b}$ values for the bleaching indications). Therefore the mean $\mathrm{L}^{*}$ values in our study increased immediately after the application of 40\% HP $\left(t_{1}\right)$ driven by it and maintained at that level over the observed period of 6 months, but the change was not significant $(\mathrm{p}>0.05)$. At the same time, mean $b^{*}$ values decreased significantly in 3 months $\left(t_{2}\right)(p<0.05)$. The reason our patients had the lighter color of the teeth was their age (around 30) and gender (most of them were women -17 participants), and it has already been proven that they are the ones who have lighter and less saturated color [23]. Our patients participated voluntarily and wanted to do the bleaching procedure because of cosmetic reasons. The results of our study confirmed that even lighter teeth could be whitened and the bleaching effect can be lasting over 6 months. One of the reasons young patients want to whiten their natural white teeth probably lies in the fact that ordinary 
light dental colors, such as A1 or A2, are not considered white enough for young patients, demanding even whiter colors. It seems that they have been influenced by the portrayal of perfect white smiles in the media [24]. It also happens because of the extremely high color quality of television, electronic devices and printed media, which have raised their self-awareness of dental color in general [24]. Therefore, the clinicians must be cautious about the patient's requests, considering the results of the whitening procedure. Another very important element is related to the bleaching procedures in young patients. Due to their accelerated, busy lives, they request the bleaching results in as little time as possible and often ask for in-office procedures. Again, it is essential for a clinician to conduct a detailed clinical examination and carefully determine the patients who meet $40 \%$ HP in-office bleaching treatment criteria.

Numerous studies have reported a similar pattern of bleaching as in our study [25-29]. Their reports found chroma influenced color changes more than lightness, with hue having almost no influence on it. The same was found in our study, too $(\mathrm{p}<0.05)$. Simultaneously, the values of Bleach index assessed using a spectrophotometer in our study decreased too, mainly during 3 months post-treatment $(\mathrm{p}<0.05)$. With the same pattern of decline as $b^{*}$ value, it can be concluded that the change in $b^{*}$ value influenced the decrease of Bleach index in this study the most. It should be mentioned that the Bleach index used in this study was digitally calculated and expressed by spectrophotometer itself and we found it the simplest clinical way to track the changes for both clinician and patient, avoiding more complicated calculations and clinical protocols used for other bleaching indices [30]. This index was designed to follow the arrangement of VITA Bleachedguide 3D-Master shade guide with the most consistent color distribution and continuous increase of lightness and decrease of chroma and vice versa [31].

The present study results are in accordance with other studies and showed that the changes in $\mathrm{L}^{*}, \mathrm{a}^{*}$ and $\mathrm{b}^{*}$ values continued long after the completion of the procedure and that the color relapse was unavoidable [21,22]. While in Al Shetheri's et al. [21] study, the color stabilized by the fifth week, in Klarić's et al. [22] after one month, in our research, it happened after 3 months.

For the color relapse, Basson et al. [32] found that it was significantly higher for the in-office treatment after a four-week or longer period. Klarić et al. [22] compared both in-office and at-home bleaching treatments and found out that $\Delta \mathrm{E}$ for at-home was lower, but in their study, the patients were using their bleaching trays one hour daily during six days in a row and used $6 \%$ HP, which is less than usual (2 weeks overnight; $18 \%$ carbamide peroxide with delayed-release). Matis et al. [11], in their review of the effectiveness of various tooth-bleaching systems, found mean $\Delta \mathrm{E}$ for overnight at-home bleaching was 9.7 immediately after the treatment, with a decreasing trend in the ten weeks after bleaching, for at-home daytime bleaching 6.6 and in-office bleaching 5.4, respectively, with a similar decreasing trend. Although the color changes in their review were lowest in in-office treatment, they were still higher than ours, but they combined the results from studies using different types in-office bleaching gels [11]. Compared to other studies investigating the effectiveness of different in-office HP gels, overall $\Delta \mathrm{E}$ in our study was relatively low.

Ontiveros and Paravina [25] used a 25\% HP in-office tooth bleaching system with and without additional light exposure and revealed a significant difference in color between treatment with light and without it (6.0 and 4.7, respectively). Although the concentration of HP in their study was lower, $\Delta \mathrm{E}$ values were higher than ours [25]. At the same time, a previous study report no influence of light on the effectiveness of the bleaching agent [33]. But different gels, treatments, assessment tools, and characteristics of the bleaching lights all contribute to the confusion associated with this topic. 
Although the overall color change in this study was lower in comparison to some studies, it was still above the 50:50\% perceptibility thresholds (PT) and 50:50\% acceptability thresholds (AT) in dentistry [33]. According to Paravina et al. [34], PT and AT were 1.22 and 2.66, respectively, and overall color change in this study was 3.6 and, therefore, $40 \%$ HP in-office bleaching treatment was considered successful.

Future studies with increased sample size having more bleaching color characteristics of the teeth to whiten (lower lightness and higher chroma assessed using spectrophotometer) and addition of other active agents, concentrations, and procedures are needed.

\section{Conclusion}

In-office bleaching treatment using $40 \%$ hydrogen peroxide was effective, and there was a statistically significant decrease in color change during the period of 6-month observation.

\section{Authors' Contributions}

$\begin{array}{lll}\text { AP } & \text { (D) https://orcid.org/0000-0001-8794-6066 } & \begin{array}{l}\text { Conceptualization, Methodology, Formal Analysis, Investigation, Data Curation, Writing - } \\ \text { Original Draft and Writing - Review and Editing. } \\ \text { Conceptualization, Methodology, Formal Analysis, Investigation and Writing - Review and } \\ \text { Editing. }\end{array}\end{array}$

All authors declare that they contributed to critical review of intellectual content and approval of the final version to be published.

\section{Financial Support}

None.

\section{Conflict of Interest}

The authors declare no conflicts of interest.

\section{Data Availability}

The data used to support the findings of this study can be made available upon request to the corresponding author.

\section{References}

[1] Joiner A, Luo W. Tooth colour and whiteness: A review. J Dent 2017; 67S:S3-S10. https://doi.org/10.1016/j.jdent.2017.09.006

[2] Goulart MA, Condessa AM, Hilgert JB, Hugo FN, Celeste RK. Concerns about dental aesthetics are associated with oral health related quality of life in Southern Brazilian adults. Cien Saude Colet 2018; 23(11):3957-64. https://doi.org/10.1590/1413-812320182311.24172016

[3] Kershaw S, Newton J, Williams D. The influence of tooth colour on the perceptions of personal characteristics among female dental patients: comparisons of unmodified, decayed and 'whitened' teeth. Br Dent J 2008; 204(5):E9; discussion 256-7. https://doi.org/10.1038/bdj.2008.134

[4] Imburgia M, Cortellini D, Valenti M. Minimally invasive vertical preparation design for ceramic veneers: a multicenter retrospective follow-up clinical study of 265 lithium disilicate veneers. Int J Esthet Dent 2019; 14(3):28698.

[5] Jankar AS, Kale Y, Pustake S, Bijjaragi S, Pustake B. Spectrophotometric study of the effect of luting agents on the resultant shade of ceramic veneers: An in vitro study. J Clin Diagn Res 2015; 9(9):ZC56-60. https://doi.org/10.7860/JCDR/2015/14377.6505

[6] Gu X, Yang L, Yang D, Gao Y, Duan X, Zhu X, et al. Esthetic improvements of postorthodontic white-spot lesions treated with resin infiltration and microabrasion: A split-mouth, randomized clinical trial. Angle Orthod 2019; 89(3):372-7. https://doi.org/10.2319/041218-274.1

[7] Knezović Zlatarić D, Žagar M, Illeš D. A clinical study assessing the short-term efficacy of combined in-office/athome bleaching treatment. J Esthet Restor Dent 2019; 31(2):140-6. https://doi.org/10.1111/jerd.12467

[8] Kothari S, Jum'ah AA, Gray AR, M Lyons K, Yap M, Brunton PA. A randomized clinical trial investigating three vital tooth bleaching protocols and associated efficacy, effectiveness and participants' satisfaction. J Dent 2020; 95:103322. https://doi.org/10.1016/j.jdent.2020.103322 
[9] Li Y, Greenwall L. Safety issues of tooth bleaching using peroxide-based materials. Br Dent J 2013; 215(1):29-34. https://doi.org/10.1038/sj.bdj.2013.629

[10] Demarco FF, Meireles SS, Masotti AS. Over-the-counter bleaching agents: a concise review. Braz Oral Res 2009; 23:64-70. https://doi.org/10.1590/s1806-83242009000500010

[11] Matis BA, Cochran MA, Eckert G. Review of the effectiveness of various tooth bleaching systems. Oper Dent 2009; 34(2):230-5. https://doi.org/10.2341/08-74

[12] Llena C, Villanueva A, Mejias E, Forner L. Bleaching efficacy of at home $16 \%$ carbamide peroxide. A long-term clinical follow-up study. J Esthet Restor Dent 2020; 32(1):12-8. https://doi.org/10.1111/jerd.12560

[13] Faus-Matoses V, Palau-Martínez I, Amengual-Lorenzo J, Faus-Matoses I, Faus-Llácer VJ. Bleaching in vital teeth: Combined treatment vs in-office treatment. J Clin Exp Dent 2019; 11(8):e754-e8. https://doi.org/10.4317/jced.56079

[14] Martins I, Onofre S, Franco N, Martins LM, Montenegro A, Arana-Gordillo LA, Reis A, et al. Effectiveness of inoffice hydrogen peroxide with two different protocols: a two-center randomized clinical trial. Oper Dent 2018; 43(4):353-1. https://doi.org/10.2341/17-128-C

[15] Marquillas CB, Procaccini R, Malmagro MV, Sánchez-Martín MJ. Breaking the rules: tooth bleaching by means of a reducing agent. Clin Oral Investig 2020; 4(8):2773-9. https://doi.org/10.1007/s00784-019-03 140-3

[16] Imbery TA, Stilianoudakis S, Tran D, Bugas CK, Seekford K. Is there an association between perceptual ability test scores and color vision acuity? J Dent Educ 2020; 84(6):688-94. https://doi.org/10.1002/jdd.12111

[17] Illeš D, Knezović Zlatarić D. Statistical power analysis for investigations using spectrophotometer VITA Easyshade ${ }^{\circledR}$ 4.0. Acta stomatol Croat 2015; 49(2):171-2.

[18] Gómez-Polo C, Gómez-Polo M, Martínez Vázquez de Parga JA, Celemín-Viñuela A. Clinical study of the 3D-master color system among the Spanish population. J Prosthodont 2018; 27(8):708-15. https://doi.org/10.1111/jopr.12584

[19] Knezović D, Zlatarić D, Illeš IŽ, Alajbeg M, Žagar. In vivo evaluations of inter-observer reliability using VITA Easyshade $^{\circledR}$ Advance 4.0 dental shade-matching device. Acta Stomatol Croat 2016; 50(1):34-9. https://doi.org/10.15644/asc50/1/5

[20] Knezović D, Zlatarić D, Illeš IŽ, Alajbeg M, Žagar. In vivo and in vitro evaluations of repeatability and accuracy of VITA Easyshade ${ }^{\circledR}$ Advance 4.0 dental shade-matching device. Acta Stomatol Croat 2015; 49(2):112-8. https://doi.org/10.15644/asc49/2/4

[21] Al Shethri S, Matis BA, Cochran MA, Zekonis R, Stropes M. A clinical evaluation of two in-office bleaching products. Oper Dent 2003; 28(5):488-95.

[22] Klarić Sever E, Budimir Z, Cerovac M, Stambuk M, Par M, Negovetic Vranic D, et al. Clinical and patient reported outcomes of bleaching effectiveness. Acta Odontol Scand 2018; 76(1):30-8. https://doi.org/10.1080/00016357.2017.1376111

[23] Hee-Kyung K. A study on the color distribution of natural teeth by age and gender in the Korean population with an intraoral spectrophotometer. J Esthet Restor Dent 2018; 30(5):408-14. https://doi.org/10.1111/jerd.12424

[24] Carey CM. Tooth bleaching: what we now know. J Evid Based Dent Pract 2014; 14 Suppl:70-6. https://doi.org/10.1016/j.jebdp.2014.02.006

[25] Ontiveros JC, Paravina RD. Color change of vital teeth exposed to bleaching performed with and without supplementary light. J Dent 2009; 37(11):840-7. https://doi.org/10.1016/j.jdent.2009.06.015

[26] Kirsten GA, Freire A, de Lima AA, Ignácio SA, Souza EM. Effect of reservoirs on gingival inflammation after home dental bleaching. Quintessence Int 2009; 40(3):195-202.

[27] Alonso de la Peña V, López Ratón M. Randomized clinical trial on the efficacy and safety of four professional at-home tooth bleaching gels. Oper Dent 2014; 39(2):136-43. https://doi.org/10.2341/12-402-C

[28] Haywood VB. Frequently asked questions about bleaching. Compend Contin Educ Dent 2003; 24(4A):324-38.

[29] Kugel G, Ferreira S, Sharma S, et al. Clinical trial assessing light enhancement of in-office tooth bleaching. J Esthet Restor Dent 2009; 21(5):226-41. https://doi.org/10.1111/j.1708-8240.2009.00287.x

[30] Pérez Mdel M, Ghine a R, Rivas MJ, Yebra A, Ionescu AM, Paravina RD, et al. Development of a customized whiteness index for dentistry based on CIELAB color space. Dent Mater 2016; 32(3):461-7. https://doi.org/10.1016/j.dental.2015.12.008

[31] Paravina R. New shade guide for tooth bleaching monitoring: visual assessment. J Prosthet Dent 2008; 99(3):178-84. https://doi.org/10.1016/So022-3913(08)60041-4

[32] Basson RA, Grobler SR, Kotze TJ, Osman Y. Guidelines for the selection of tooth bleaching products amongst those available on the market. SADJ 2013; 68(3):122-9.

[33] Hein DK, Ploeger BJ, Hartup JK, Wagstaff RS, Palmer TM, Hansen LD. In-office vital tooth bleaching - what do lights add? Compend Contin Educ Dent 2003; 24(4A):340-52.

[34] Paravina RD, Ghinea R, Herrera LJ, Bona AD, Igiel C, Linninger M, et al. Color difference thresholds in dentistry. J Esthet Restor Dent 2015; 27: S1-9. 